The Astrophysical Journal, 213:38-42, 1977 April 1

(c) 1977. The American Astronomical Society. All rights reserved. Printed in U.S.A.

\title{
KINEMATICS OF CARBON MONOXIDE OBSERVED WITHIN ONE DEGREE OF THE GALACTIC CENTER
}

\author{
H. S. Liszt AND W. B. Burton \\ National Radio Astronomy Observatory,* Green Bank, West Virginia \\ R. H. SANDERS \\ University of Pittsburgh \\ AND \\ N. Z. SCOVILLE \\ University of Massachusetts \\ Received 1976 August 27
}

\begin{abstract}
Observations of the inner $2^{\circ}$ of galactic longitude have been made in the $2.6 \mathrm{~mm}$ transition of carbon monoxide with $1^{\prime}-2^{\prime}$ beam spacings. The resulting maps show several large scale $(>100 \mathrm{pc}$ ) patterns which correlate well with features present in $\mathrm{H} \mathbf{I}$, including the nuclear disk and the $3 \mathrm{kpc}$ arm. Several features are identified with absorption by very cold foreground clouds; the $3 \mathrm{kpc}$ arm feature occurs in both emission and absorption.
\end{abstract}

Subject headings: galaxies: Milky Way - galaxies: nuclei - galaxies: structure interstellar: molecules

\section{INTRODUCTION}

The $2.6 \mathrm{~mm}$ spectral line of carbon monoxide is especially useful for investigation of the nuclear region of the Galaxy because of the general transparency of the interstellar medium at $2.6 \mathrm{~mm}$, because of the high angular resolution afforded by the short wavelength, and because of the relative strength and ubiquity of the carbon monoxide emission. Other investigations of the nuclear region have not, for practical reasons, combined high spatial and spectral resolution with wide longitude coverage. The present observations represent an improvement in this respect, covering the inner $2^{\circ}$ of galactic longitude with high spatial resolution $\left(1^{\prime}\right.$ to $2^{\prime}$, corresponding at $R_{0}=10 \mathrm{kpc}$ to 3 to $6 \mathrm{pc}$ ) and with high spectral resolution $\left(2.6 \mathrm{~km} \mathrm{~s}^{-1}\right)$.

Although some of the structural aspects of the galactic nucleus found in the present observations were also seen in earlier $\mathrm{cm}$ - and $\mathrm{mm}$-wavelength investigations, the overall gas distribution can now be traced much more easily. Several of the most prominent features show motions which are often attributed predominantly to rotation. One such feature, identified here for the first time, is the molecular counterpart of the "pure rotation" nuclear disk component which is so prominent in atomic hydrogen. A further consequence of the new observations is the presence of several carbon monoxide absorption features, suggesting that extremely cold $(\sim 3 \mathrm{~K})$ and, in general, undetectable molecular clouds occur rather commonly in the Galaxy. A feature identified as the $3 \mathrm{kpc}$ arm is visible both in emission and absorption.

* Operated by Associated Universities, Inc., under contract with the National Science Foundation.

\section{OBSERVATIONS}

The observations were made during 1975 May and July at the 36 foot $(11 \mathrm{~m})$ radio telescope of the National Radio Astronomy Observatory on Kitt Peak. The single-sideband noise temperature of the cooled-mixer receiver was $\sim 800$ or $\sim 1500 \mathrm{~K}$ (including the atmosphere), with the larger value occurring during July. All observations were taken through clear skies. The filter bank consisted of 256 channels, each of $1 \mathrm{MHz}\left(2.6 \mathrm{~km} \mathrm{~s}^{-1}\right)$ width and separated by $1 \mathrm{MHz}$ : the total velocity coverage of $650 \mathrm{~km} \mathrm{~s}^{-1}$ is sufficiently large to include all known emission from the nuclear region. Because no matching of partial spectra was required, the observations are quite sensitive to broad, weak features. We observed in the position-switching mode by moving the telescope during each observation (of 10 minutes duration) to a reference position at $\alpha, \delta(1950)=17^{\mathrm{h}} 34^{\mathrm{m}} 39^{\mathrm{s}}$, $-26^{\circ} 29^{\prime} 17^{\prime \prime}$. This direction was itself carefully observed and determined to be free of $\mathrm{CO}$ emission. In spite of the sensitivity of the cooled receiver to changes in elevation during the position-switching cycle, the baselines of the profiles were generally quite flat. In a few cases, linear baselines were subtracted from an entire spectrum.

We observed along several strips of longitude, generally at $1^{\prime}$ intervals at $|\Delta l| \leq 10^{\prime}$, and at either $2^{\prime}$ or $5^{\prime}$ intervals outside this range. The displacements $\Delta l$ and $\Delta b$ are measured with respect to the peak in $2 \mu \mathrm{m}$ radiation in the direction of Sgr A (West) at $l=-00^{\circ} 03^{\prime} 20^{\prime \prime}, b=-00^{\circ} 02^{\prime} 45^{\prime \prime}$ (Rieke and Low 1973). This position evidently corresponds to that of the peak of the distribution of normal stars, and thus to the direction of the dynamical center of the Galaxy. The 


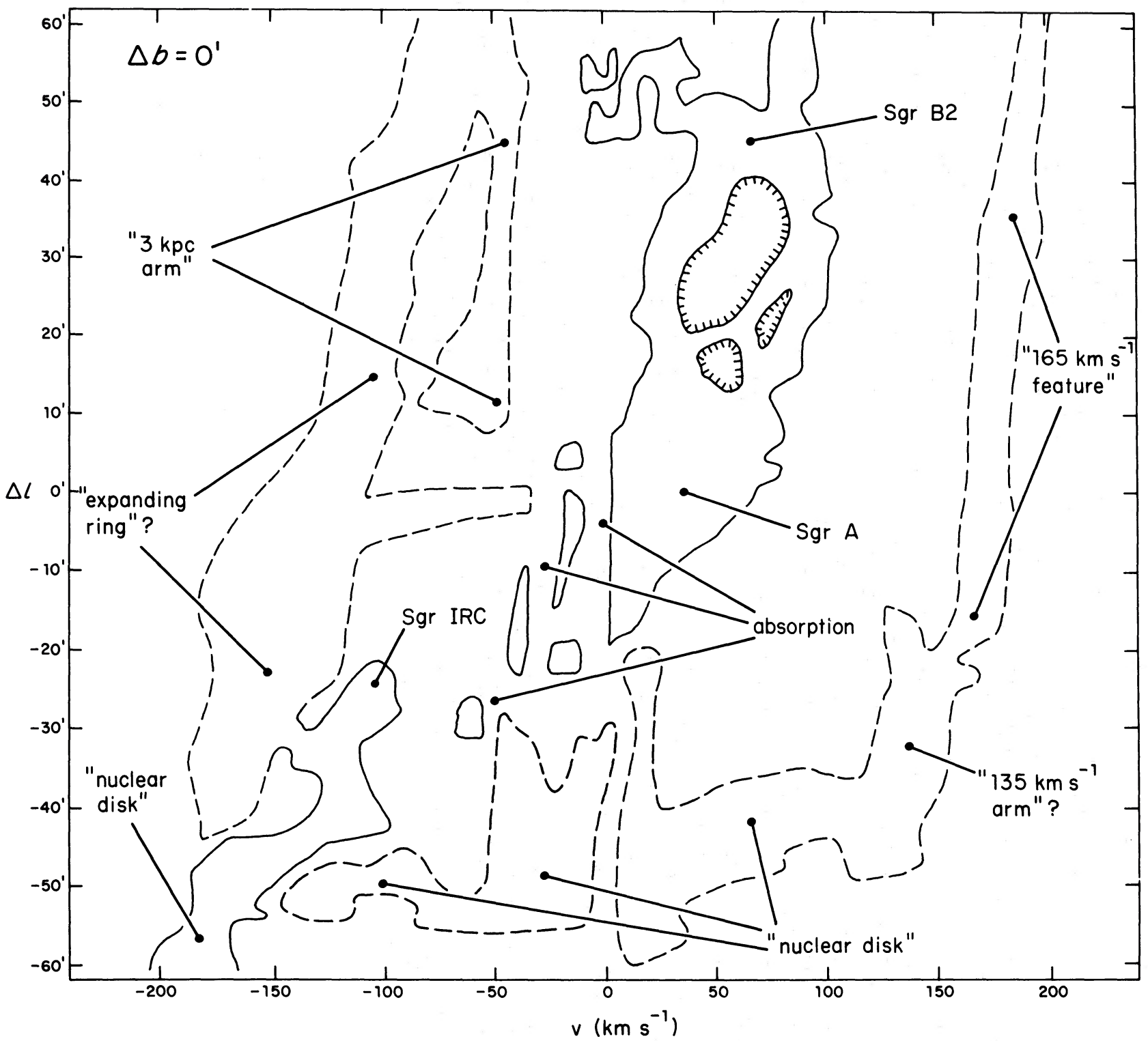

FIG. 1b.-Schematic representation of the longitude-velocity arrangement characterizing the observations entering Fig. 1a. The full-drawn contours outline the more intense CO features; the dashed contours outline less intense emission features.

longitude-velocity distribution of the observed $\mathrm{CO}$ intensities are shown in Figures $1 a$ and 2-5 (Plates 3-7). The gray-scale representation of intensities is such that the perceived brightness scale varies linearly with the observed intensities, except at the highest levels where some structure has been lost during the photographic process. It is our experience that these gray-scale representations, made with the NRAO Dicomed image processor, greatly facilitate locating kinematic patterns in the observations. The major kinematic patterns identified in the observations at $\Delta b=0^{\prime}$ (Fig. 1a) are labeled in the schematic representation in Figure $1 b$. All velocities refer to the local standard of rest.

\section{DISCUSSION OF THE OBSERVATIONS}

Several general conclusions are apparent from inspection of the figures and will be discussed below.

1. If the dynamical center of the Galaxy is near the direction $\Delta l=0^{\prime}$, the great majority of the observed intensity occurs at velocities which are compatible with galactic rotation.

2. Four classes of gas can be usefully distinguished in the observations; only the latter two are physically associated with the galactic nucleus.

i) Very cold gas appearing in absorption at $v \approx 0,-30$, and $-55 \mathrm{~km} \mathrm{~s}^{-1}$. The $-55 \mathrm{~km} \mathrm{~s}^{-1}$ feature is part of the $3 \mathrm{kpc}$ arm. 
ii) Low-intensity gas emitting at $|v| \preccurlyeq 10 \mathrm{~km} \mathrm{~s}^{-1}$, characterized by the temperatures, dispersions, and velocities found typical for $\mathrm{CO}$ clouds distributed throughout the inner $8 \mathrm{kpc}$ of the Galaxy (Scoville and Solomon 1975; Burton et al. 1975; Gordon and Burton 1976) and accumulated near $\Delta l=0^{\circ}$ over lengths of path of order $10 \mathrm{kpc}$.

iii) High-intensity gas (outlined by full-drawn lines in Fig. 1b), almost all of which occurs either in the quadrant $\Delta l<0^{\prime}, v<0 \mathrm{~km} \mathrm{~s}^{-1}$ or in the quadrant $\Delta l>0^{\prime}, v>0 \mathrm{~km} \mathrm{~s}^{-1}$.

iv) Low-intensity gas (outlined by dashed lines in Fig. 1b), occurring at large velocities throughout the $(\Delta l, v)$-plane and indicating highly noncircular velocities.

3. It is possible to trace kinematic patterns throughout the observed region. The pattern of the highestintensity gas crosses $v=0 \mathrm{~km} \mathrm{~s}^{-1}$ near $\Delta l=0^{\prime}$.

4. The low-intensity gas at high negative velocities is more prominent at lower latitudes. The same situation holds for the stronger features near the direction of Sgr A. The distribution of low-intensity gas at high positive velocities is tilted with respect to the plane $\Delta b=0^{\prime}$ in the sense that emission at positive (negative) longitudes is more intense at lower (higher) latitudes.

\section{a) Features Appearing in Absorption}

Common to the observations in all of the figures is the presence of several bands of anomalously low intensity, sharply defined through their narrowness $\left(\$ 10 \mathrm{~km} \mathrm{~s}^{-1}\right)$, and extending vertically over large portions of the $(\Delta l, v)$-plane. We identify these as being due to absorption by cold $\mathrm{CO}$ gas located somewhere on the line of sight between the Sun and the galactic center. With regard to this identification, we stress the care with which we determined the reference position to be emission-free. Around Sgr A, absorption near $v=0 \mathrm{~km} \mathrm{~s}^{-1}$ is also present in observations of the ${ }^{13} \mathrm{CO}$ isotope line (Liszt, Sanders, and Burton 1975), thus providing a lower limit, $10^{18} \mathrm{~cm}^{-2}$, to the column density of absorbing $\mathrm{CO}$. With characteristic dimensions of $30^{\prime}(\Delta l)$ and $>8^{\prime}(\Delta b)$, this one obscuring cloud alone contains more than $10^{4} M_{\odot}$ if it is as distant as $1 \mathrm{kpc}$. Such very cold clouds may contain a significant portion of the mass of the interstellar medium, because it is unlikely that they are limited to the direction of the galactic center. This direction merely provides the most (if not the only) suitable mm-wave background contribution allowing positive identification of the cold gas. In several of the figures the $3 \mathrm{kpc}$ arm feature is seen in emission at $\Delta l \geqslant-10^{\prime}$, but in absorption at lower longitudes.

The measured intensities in these features sometimes reach $\sim 0 \mathrm{~K}$ in the center of the lines, implying that the kinetic temperature of the absorbing gas is $\sim 3 \mathrm{~K}$. Although the physical conditions in molecular clouds are imperfectly understood at present, it is nevertheless remarkable that the optically thick absorbing clouds do not appear to be temperature-coupled to the ambient gas. It will be interesting to see in future observations whether other constituents of the inter- stellar gas normally observed in emission also show absorption characteristics analogous to those shown by carbon monoxide. We note that observations of $\mathrm{NH}_{3}$ by Kaifu et al. (1975) show zero-velocity emission at $\Delta l \sim 10^{\prime}$ and $\sim 30^{\prime}$, where $\mathrm{CO}$ absorption is present.

\section{b) Features Compatible with Pure Rotation}

Earlier analyses of molecular observations of the galactic nucleus showed little or no evidence for kinematic patterns with the characteristic longitudevelocity arrangement identified with galactic rotation. The prototype rotation signature is displayed by the well-known nuclear disk component found in observations of atomic hydrogen. Although the $\mathrm{CO}$ distribution observed here is complicated by the presence of absorption features and, at $\Delta l>0$, by the presence of the Sgr A and Sgr B sources, we believe that this simple signature does appear prominently in molecular gas kinematics. In Figure $1 a$, emission can be traced from $(\Delta l, v)=\left(-50^{\prime}, \quad-160 \mathrm{~km} \mathrm{~s}^{-1}\right)$ to $\left(-30^{\prime}\right.$, $\left.-100 \mathrm{~km} \mathrm{~s}^{-1}\right)$, through two or more absorption features to $\left(-15^{\prime}, 35 \mathrm{~km} \mathrm{~s}^{-1}\right)$ and $\left(-4^{\prime},-15 \mathrm{~km} \mathrm{~s}^{-1}\right)$, across $\left(\sim 0^{\prime}, \sim 0 \mathrm{~km} \mathrm{~s}^{-1}\right)$ to $\left(+10^{\prime},+60 \mathrm{~km} \mathrm{~s}^{-1}\right)$, $\left(+20^{\prime},+80 \mathrm{~km} \mathrm{~s}^{-1}\right)$ and $\left(+30,+95 \mathrm{~km} \mathrm{~s}^{-1}\right)$. Beyond $\Delta l \sim 30^{\prime}$ the presence of Sgr B complicates the appearance of the spectra. A single kinematic feature is thus fairly well defined over some $250 \mathrm{pc}$ and $250 \mathrm{~km}$ $\mathrm{s}^{-1}$, and over both positive and negative longitudes and velocities. For $\Delta l<0, v<0$, this pattern corresponds to the strong ridge present in the $21 \mathrm{~cm}$ line (cf. Wrixon and Sanders 1973). When the present data are combined with $\mathrm{CO}$ observations at $|l|>1^{\circ}$ (see Bania 1976 and Scoville, Solomon, and Jefferts 1974), the continuity of this rotation-component is all the more pronounced. At positive longitudes, the bridge of emission between Sgr A and B which was first discussed by Solomon et al. (1972) is apparently an integral part of a much larger structure, which at $|\Delta l| \leq 30^{\prime}$ shows a high degree of symmetry about $\left(\sim 0^{\prime}, 0 \mathrm{~km} \mathrm{~s}^{-1}\right)$. At $\Delta l<-50^{\prime}$, the high negative velocity gas could be associated with either the highor low-intensity gas populations, but it is possible (and suggested especially at $\Delta b=0^{\prime}$ ) to trace the rotation out to $\sim-190 \mathrm{~km} \mathrm{~s}^{-1}$ at $\Delta l \sim-60^{\prime}$.

Observations at $1^{\prime}$ beam-spacings around the direction of Sgr A indicate that the molecular gas crosses from negative to positive velocities at slightly negative $\Delta l$ (see Fig. 2 at $\Delta b=-2^{\prime}$ ). At $\Delta l=0^{\prime}$, the dominant emission occurs at $v=35 \mathrm{~km} \mathrm{~s}^{-1}$, and it is not until $\Delta l \sim-2^{\prime}$ that it begins to disappear behind the zerovelocity absorption feature. At $\Delta l \sim-6^{\prime}$ it emerges again at negative velocities. Because small noncircular velocity components due to random motions are especially apparent near $\Delta l \sim 0^{\prime}$, our observations are probably consistent with placement of the dynamical center of the Galaxy at or very near the direction of Sgr A (West).

c) The Sagittarius Sources

The detailed structure of the molecular clouds observed toward Sgr A and B has been the subject of 
several recent investigations. Here, we consider the relationship of these two powerful but localized sources to the distributed gas.

\section{i) $\operatorname{Sgr} A$}

In the immediate vicinity of Sgr A, kinematic components are seen at $v=20$ and $v=60 \mathrm{~km} \mathrm{~s}^{-1}$ (Solomon et al. 1972; Liszt, Sanders, and Burton 1975). At the position of Sgr A (West), the $20 \mathrm{~km} \mathrm{~s}^{-1} \mathrm{CO}$ and $\mathrm{H}_{2} \mathrm{CO}$ line strengths reach a local minimum; from this behavior, Liszt, Sanders and Burton (1975) argued that Sgr A (East) is not necessarily behind Sgr A (West), as $6 \mathrm{~cm}$ interferometry in the $\mathrm{H}_{2} \mathrm{CO}$ line might otherwise indicate (Whiteoak, Rogstad, and Lockhart 1974). Aperture synthesis of $\mathrm{OH}$ absorption seems to support this contention (Bieging 1976). Perhaps more important from the point of view of the present discussion is the fact that the $\mathrm{CO}$ emission and $\mathrm{H}_{2} \mathrm{CO}$ absorption have identical kinematic behavior between 0 and $80 \mathrm{~km} \mathrm{~s}^{-1}$ and therefore evidently arise from the same volume of space.

The strongest molecular emission in the vicinity of Sgr A lies mainly at $\Delta b \leq 0^{\prime}$ : the gas distribution may also be tilted with respect to the plane $\Delta b=0^{\prime}$ because $20 \mathrm{~km} \mathrm{~s}^{-1}$ emission is concentrated to lower latitudes. The observations show that the exact position of Sgr A (West) is rather unremarkable, and that the kinematics around this source are dominated by the large velocity gradient of a very widespread gas component. Both "Sgr A" components, at 20 and $60 \mathrm{~km} \mathrm{~s}^{-1}$, are clearly extended over the $45^{\prime}$ of longitude between Sgr A and B, and neither can properly be identified with local behavior at the center of the Galaxy.

\section{ii) $\operatorname{Sgr} B$}

At longitudes $l \geqslant 30^{\prime}$, the large, nearly constant velocity gradient observed at more negative longitudes disappears completely as the gap between the two bands of the emission bridge is filled in by structure associated with the Sgr B molecular cloud. Scoville, Solomon, and Penzias (1975) have shown that line profiles of many molecular species observed toward Sgr $\mathrm{B}_{2}(\mathrm{OH})$ are dependent on the optical depth of the transition studied in the sense that optically thick transitions have an intensity minimum at $v=$ $62 \mathrm{~km} \mathrm{~s}^{-1}$ but those that are thin have an intensity maximum. Although this is a strong argument for self-reversal in the optically thick lines, it is interesting to note that the kinematic behavior of the emission intensity of the optically thick CS $J=2-1$ transition is identical to that of the optical depth in $6 \mathrm{~cm} \mathrm{H}_{2} \mathrm{CO}$ (Rogstad, Lockhart, and Whiteoak 1974) and that absorption measurements in $\mathrm{H}_{2} \mathrm{CO}$ and $\mathrm{OH}$ clearly point to the presence of a distinct $80 \mathrm{~km} \mathrm{~s}^{-1}$ component very near the Sgr B continuum sources. The distributed (larger scale) gas at $90 \mathrm{~km} \mathrm{~s}^{-1}$ evidently, according to the absorption measurements of Scoville et al. (1972), lies behind Sgr B: nevertheless, our CO maps show a clear tendency for emission from this gas to increase in intensity near the continuum sources, implying that Sgr B simply lies near the proximal edge of the larger feature. (See the schematic drawing in Scoville 1972 where Sgr B was placed just inside his molecular ring.) It seems an unlikely coincidence that Sgr B should occur so close to the region where the overall pattern of the distributed gas suddenly changes character. Although the observational situation is not entirely obvious, it does appear that the kinematic situation in the vicinity of Sgr B is fundamentally different from that pertaining nearer the center of the Galaxy.

\section{d) Features Characterized by High, Obviously Noncircular Velocities}

Around the borders of the longitude-velocity diagram at $\Delta b=0^{\prime}$ is a horseshoe shaped locus of lowintensity emission with $|v| \sim 130-170 \mathrm{~km} \mathrm{~s}^{-1}$ at $\Delta l \sim 0^{\prime}$ (dashed outlines in Fig. 1b). This structure, or various parts of it, have been discussed by Scoville (1972), Solomon et al. (1972), Scoville, Solomon, and Jefferts (1974), Sanders and Wrixon (1973), and Kaifu, Iguchi, and Kato (1974). The original molecular ring models were constructed in order to account for the wide kinematic structure at $\Delta l \sim-50^{\prime}$, all the high negative velocity, low-intensity gas, and the emission peak at $\Delta l \sim 56^{\prime}, v \sim+85 \mathrm{~km} \mathrm{~s}^{-1}$. At positive velocities and $|\Delta l| \leqslant 50^{\prime}$ there are no observed features corresponding to some $40 \%$ of the proposed ring. To construct the models, a connection was drawn across zero velocity at $\Delta l \sim 50^{\prime}$, tracing emission which is certainly due to some extent to the (unrelated) $3 \mathrm{kpc}$ arm. The true longitude extent of the high negative velocity gas is actually quite difficult to determine. At positive longitudes, the observed velocity gradient of $1 \mathrm{~km} \mathrm{~s}^{-1}$ arcminute $^{-1}$ carried it toward zero velocity where it becomes confused with other features. At negative longitudes, it becomes blended at $\Delta l \sim-40^{\prime}$ and may either cross zero velocity at $\Delta l \sim-50^{\prime}$ (Fig. $1 a$ at $\Delta b=0^{\prime}$ ) or continue to still more negative longitude (Fig. 3 at $\Delta b=4^{\prime}$ ). The latitude distribution is more easily gleaned from the observations, as they simply show the intensity of this gas increasing at lower latitudes.

At all latitudes, the high positive velocity gas is seen to terminate near $\Delta l=-50^{\prime}$, where a broad feature stretches from $v \approx-150$ and $+150 \mathrm{~km} \mathrm{~s}^{-1}$ (at least at $\Delta b=0^{\prime}$; at $\Delta b= \pm 4^{\prime}$, only the positive velocities are seen). No analogous crossing of zero velocity is present at positive longitudes, higher than those observed here, on the maps of Scoville, Solomon, and Jefferts (1974) and of Bania (1976). The intensity distribution of the high positive velocity gas is tilted with respect to the plane $\Delta b=0^{\prime}$, in the sense that emission at more positive (negative) longitude is more prominent at lower (higher) latitude. This behavior mimics that of the $+135 \mathrm{~km} \mathrm{~s}^{-1}$ expanding arm seen at $21 \mathrm{~cm}$ (Sanders and Wrixon 1973) and for much the same reason: the prominence at zero or positive latitude and $-40^{\prime}<\Delta l<-20^{\prime}$ of a feature at $+135 \mathrm{~km} \mathrm{~s}^{-1}$. When the $21 \mathrm{~cm}$ line is observed with sufficiently high resolution $\left(9^{\prime}\right.$; Wrixon and Sanders 
1976), a ridge is clearly visible analogous to that observed in the $\mathrm{CO}$ emission at $v=+165 \mathrm{~km} \mathrm{~s}^{-1}$ and $\Delta l \approx 0^{\prime}$.

The broad emission at $-50^{\prime}<\Delta l<-40^{\prime}$ covering the velocity interval $|v| \leq 50 \mathrm{~km} \mathrm{~s}^{-1}$ has several possible explanations. At $\Delta b=0^{\prime}$ there is a definite suggestion that the high negative velocity gas crosses $v=0$ near $\Delta l=-50$, and that it is occulted by "nuclear disk" emission when $-50^{\prime} \leq \Delta l \leq-40^{\prime}$. This interpretation has the virtue that it connects together all of the gas exhibiting high noncircular motion, but with the unsatisfying aspect that the $165 \mathrm{~km} \mathrm{~s}^{-1}$ feature extends to $\Delta l \geq 2^{\circ}$ without recrossing zero velocity. Alternatively, when one views our data in the context of coarser maps covering a wider range in latitude, the broad emission at $\Delta l \sim-50^{\prime}$ appears to be part of a remarkably symmetric body covering the entire interval $|\Delta l| \leqslant 2.5^{\circ}$. The "nuclear disk" and $165 \mathrm{~km} \mathrm{~s}^{-1}$ feature seem to radiate from the broad emission at nearly right angles in the $(\Delta l, v)$-plane, with nearly perfect symmetry about $\Delta l \sim-50^{\prime}, v \sim 0$. Such an explanation requires the existence of a new center of symmetry displaced from the dynamical center by $\sim 150 \mathrm{pc}$, and associates emission from the nuclear disk with components having high noncircular motions.

\section{SUMMARY}

Over some $90^{\prime}$ of longitude, corresponding to a linear scale of about $250 \mathrm{pc}$, a pattern seen in $\mathrm{H}$ I and usually attributed to pure rotation can explain the kinematics of a major part of the observations of molecular clouds associated with the galactic nucleus. The more positive velocity arm of the emission bridge between Sgr B and Sgr A, the gas observed toward Sgr A, and emission corresponding to the intense $21 \mathrm{~cm}$ ridge at negative longitudes all exhibit this characteristic pattern. At several velocities, the galactic center gas is obscured by absorption due to cold, foreground clouds. Near the position and velocity of Sgr B, the rotation signature is severely distorted, creating a marked asymmetry about zero longitude and velocity. At all longitudes considered here, low intensity gas is present at large negative and positive velocities $\left(|v| \sim 130-200 \mathrm{~km} \mathrm{~s}^{-1}\right)$, which are forbidden in the sense of galactic rotation.

In none of the models proposed by earlier authors for molecular cloud kinematics near the galactic nucleus did pure rotation play a significant role. This situation arose because of apparent dissimilarities in the patterns traced by molecular and atomic gas. Nonetheless, when these two gas constituents are observed with sufficiently high resolution, they begin to resemble each other more and more strongly. Using molecular emission to examine the inner few degrees of longitude (which are difficult to study in $\mathrm{H}$ I because of absorption) and atomic hydrogen beyond, it should be seen to be possible to unify the kinematics of all the gas in the inner Galaxy. Such a study is now in progress.

\section{REFERENCES}

Bania, T. M. 1976 (in preparation).

Bieging, H. 1976, submitted to Astr. and Ap.

Burton, W. B., Gordon, M. A., Bania, T. M., and Lockman, F. J. 1975, Ap. J., 202, 30.

Gordon, M. A., and Burton, W. B. 1976, Ap. J., 208, 346.

Kaifu, N., Iguchi, T., and Kato, T. 1974, Publ. Astr. Soc. Japan, 26, 117.

Kaifu, N., Morris, M., Palmer, P., and Zuckerman, B. 1975, Ap.J., 201, 98.

Liszt, H. S., Sanders, R. H., and Burton, W. B. 1975, Ap. J., 198, 537.

Rieke, G. H., and Low, F. J. 1973, Ap. J., 184, 415.

Rogstad, D. H., Lockhart, I. A., and Whiteoak, J. B. 1974, Astr. Ap., 36, 253.

Sanders, R. H., and Wrixon, G. T. 1973, Astr. Ap., 26, 365.

Scoville, N. Z. 1972, Ap. J. (Letters), 175, L127.

Scoville, N. Z., and Solomon, P. M. 1975, Ap. J. (Letters), 199, L105.

Scoville, N. Z., Solomon, P. M., and Jefferts, K. B. 1974, Ap. J. (Letters), 187, L63.

Scoville, N. Z., Solomon, P. M., and Penzias, A. A. 1975, Ap. J., 201, 352.

Solomon, P. M., Scoville, N. Z., Jefferts, K. B., Penzias, A. A., and Wilson, R. W. 1972, Ap. J., 178, 125.

Whiteoak, J. B., Rogstad, D. H., and Lockhart, I. A. 1974, Astr. Ap., 36, 245.

Wrixon, G. T., and Sanders, R. H. 1976 (in preparation).

W. B. BurTon and H. S. Liszt: National Radio Astronomy Observatory, Edgemont Road, Charlottesville, VA 22901

R. H. SANDERs: Department of Physics, University of Pittsburgh, Pittsburgh, PA 19081

N. Z. ScovilLE: Department of Physics and Astronomy, University of Massachusetts, Amherst, MA 01002 
PLATE 3

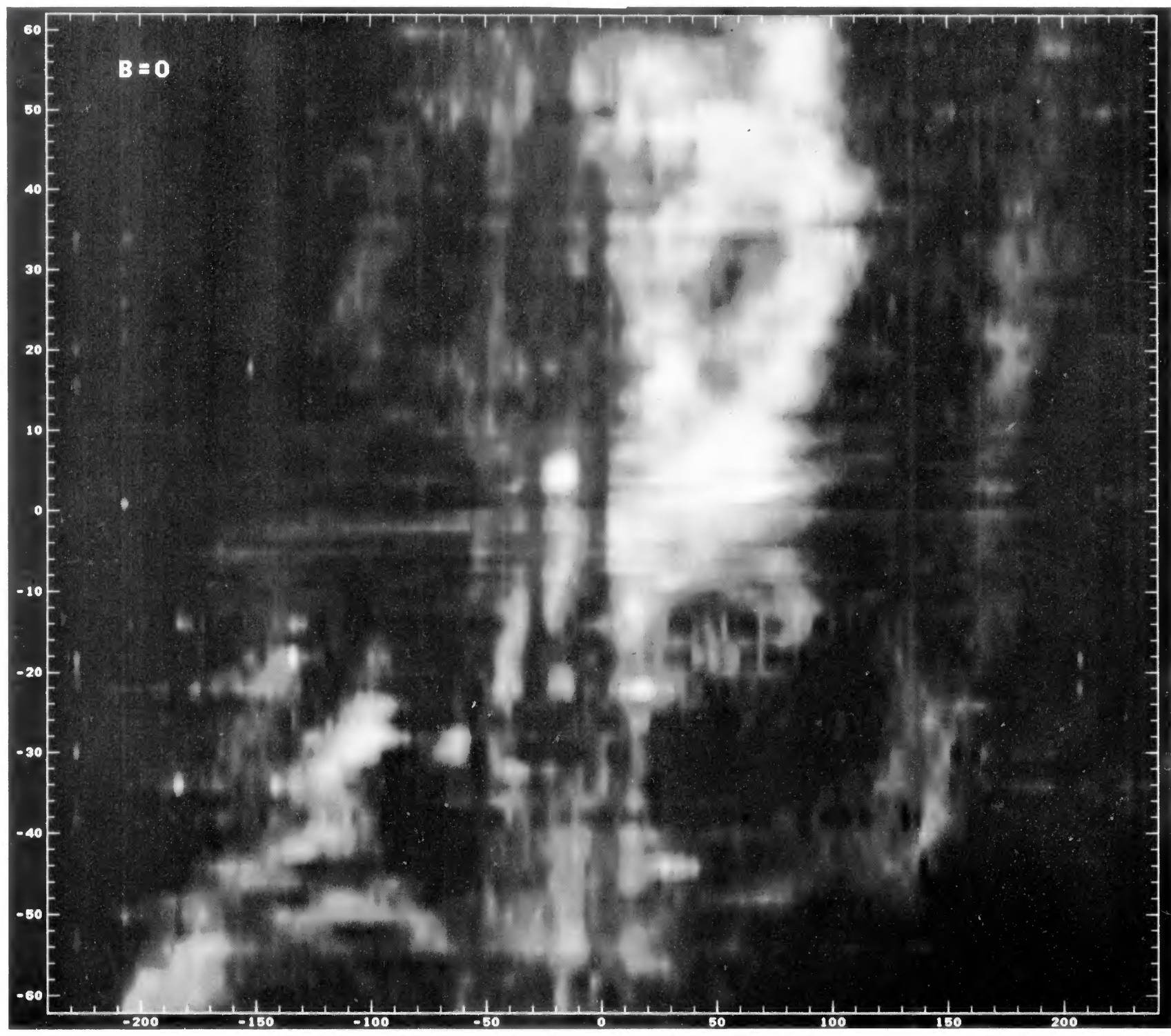

Frg. $1 a$ - Gray-scale representation of ${ }^{12} \mathrm{C}^{16} \mathrm{O}$ emission and absorption intensities in longitude-velocity coordinates at $\Delta b=0{ }^{\prime}$ $-62^{\prime} \leq \Delta l \leq 62^{\prime}$. At $|\Delta l|<10^{\prime}$, the observations are separated in longitude by $1^{\prime}$; elsewhere by $2^{\prime}$. $\Delta l$ and $\Delta b$ are measured with respect to $l=-00^{\circ} 03^{\prime} 20^{\prime \prime}$ and $b=-00^{\circ} 02^{\prime} 45^{\prime \prime}$, respectively.

LiszT et al. (see page 39) 
PLATE 4

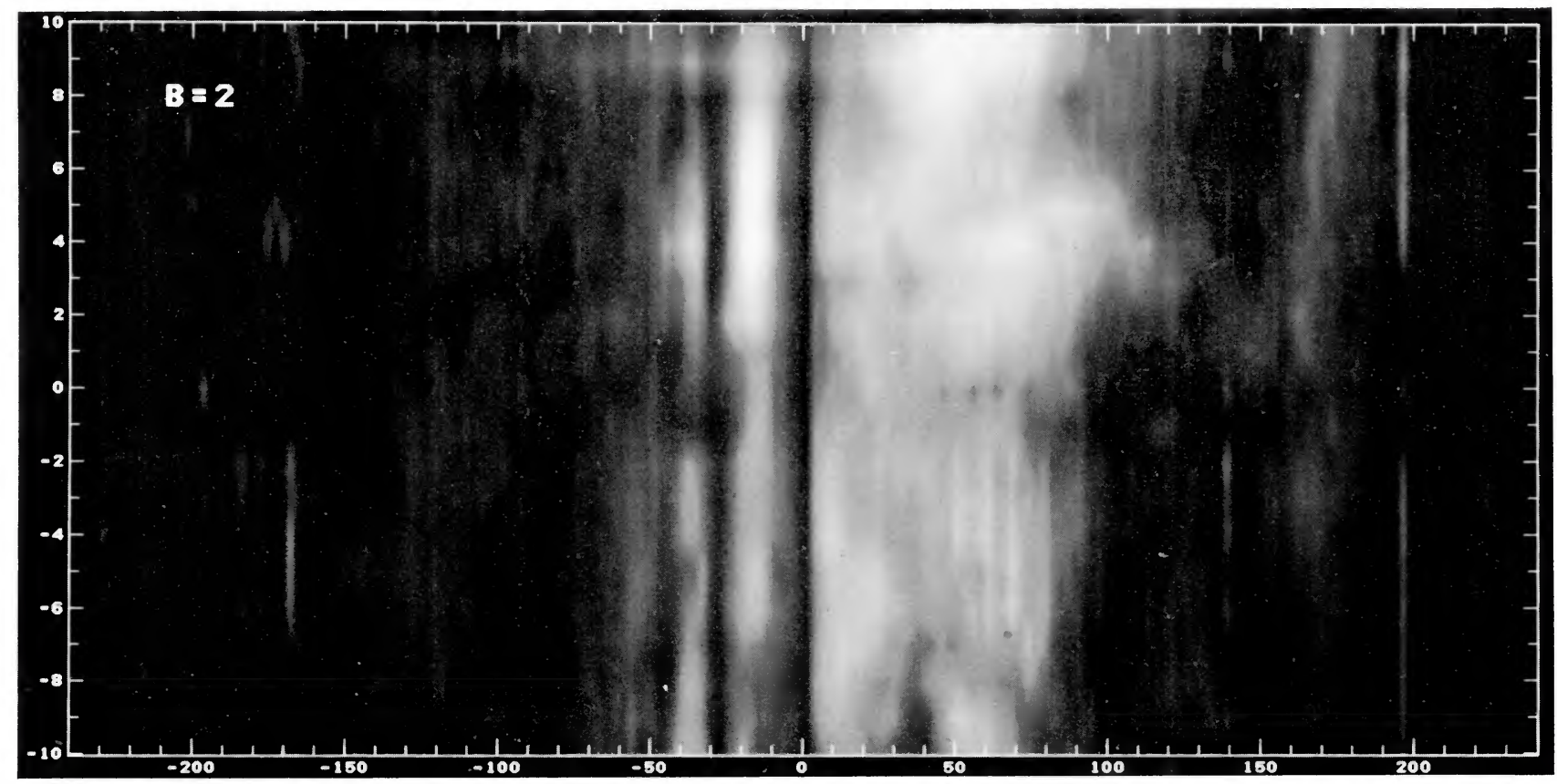

FIG. 2.-Gray-scale representation of ${ }^{12} \mathrm{C}^{16} \mathrm{O}$ emission and absorption intensities in longitude-velocity coordinates at $\Delta b=2$, $-10^{\prime} \leq \Delta l \leq 10^{\prime}$. The observations are separated in longitude by $1^{\prime}$.

LiszT et al. (see page 39) 
PLATE 5

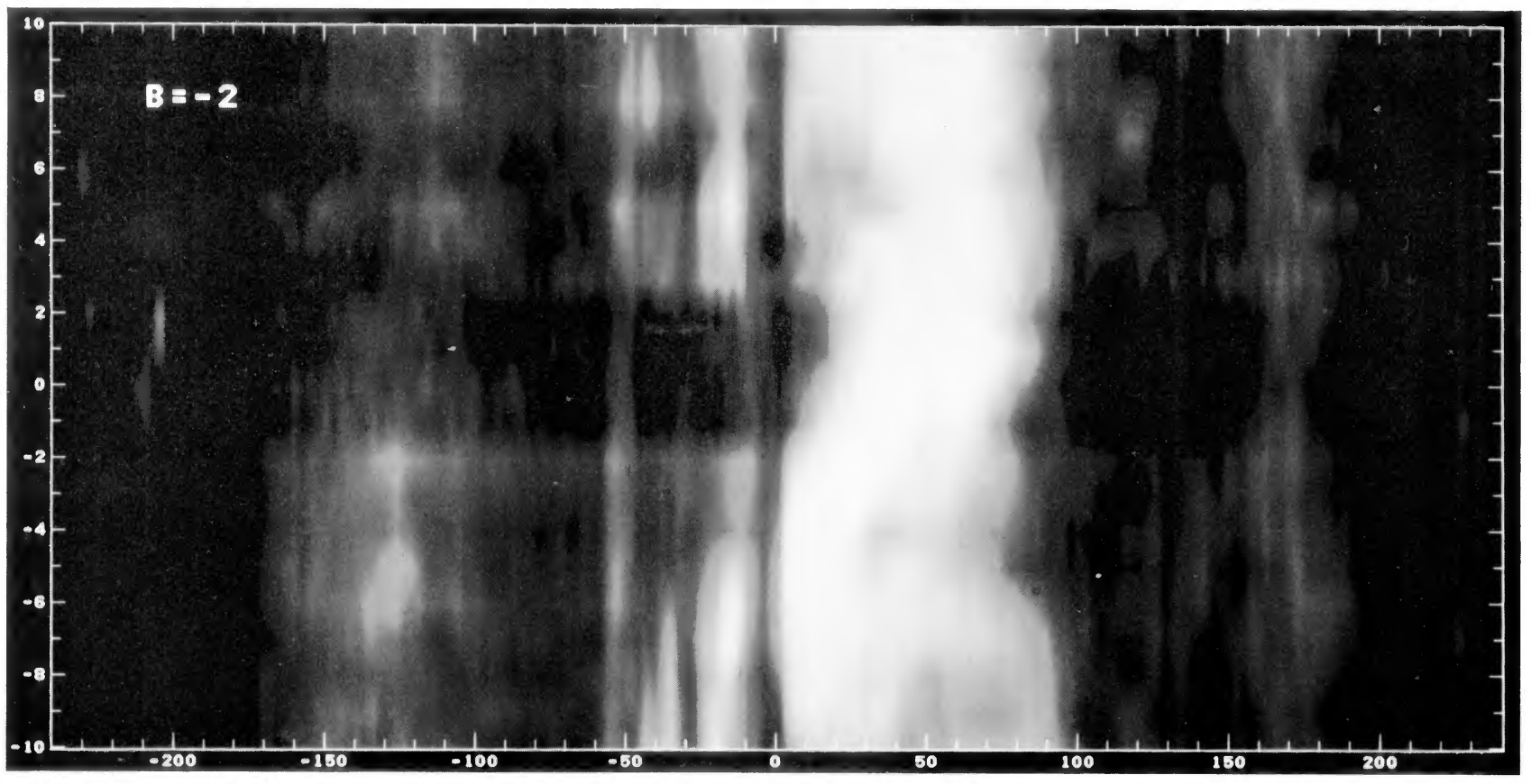

FIG. 3.-Gray-scale representation of ${ }^{12} \mathrm{C}^{16} \mathrm{O}$ emission and absorption intensities in longitude-velocity coordinates at $\Delta b=-2^{\prime}$, $-10^{\prime} \leq \Delta l \leq 10^{\prime}$. The observations are separated in longitude by $1^{\prime}$.

LiszT et al. (see page 39) 
PLATE 6

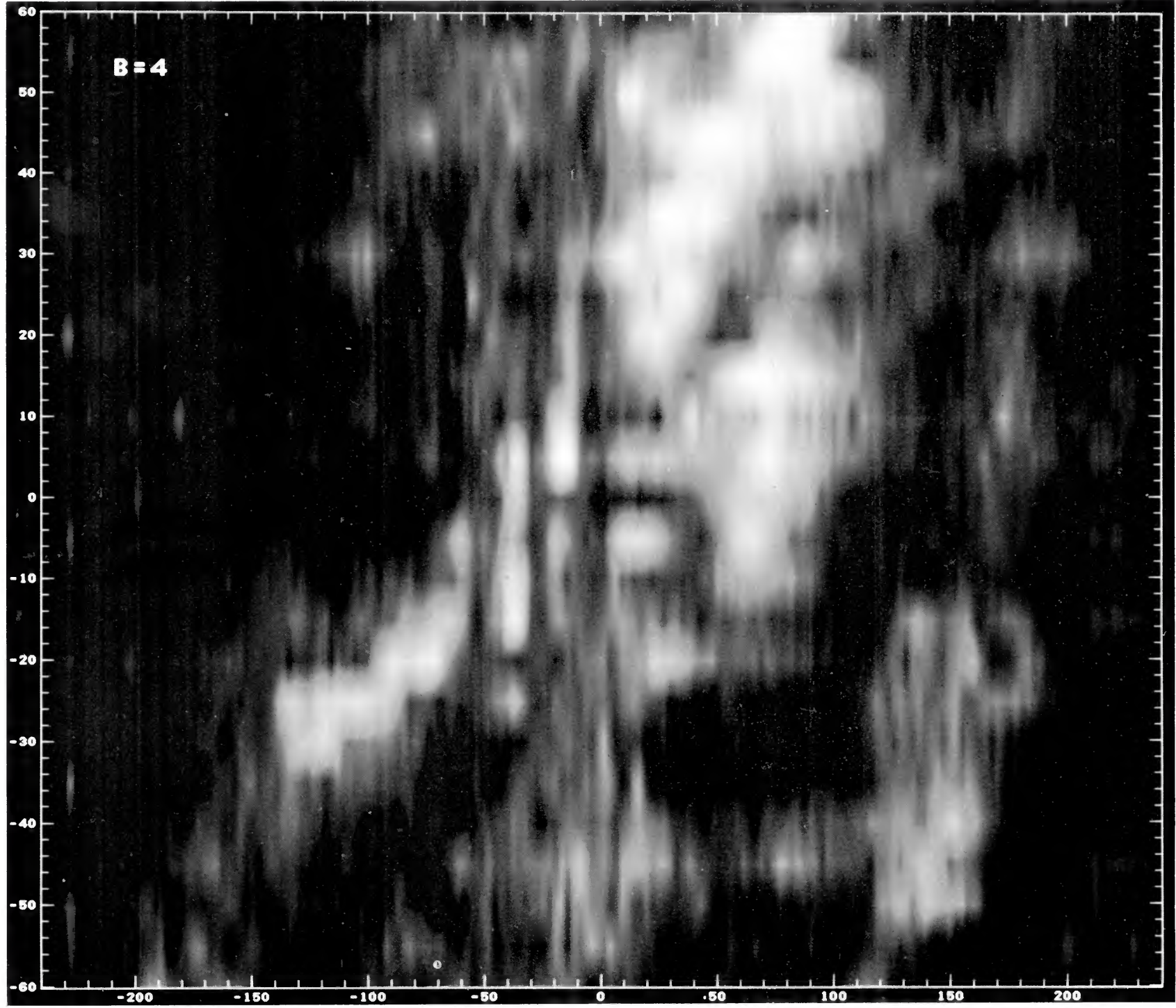

FIG. 4.-Gray-scale representation of ${ }^{12} \mathrm{C}^{16} \mathrm{O}$ emission and absorption intensities in longitude-velocity coordinates at $\Delta b=4^{\prime}$, $-60^{\prime} \leq \Delta l \leq 60^{\prime}$. The observations are separated in longitude by $5^{\prime}$.

Liszt et al. (see page 39) 


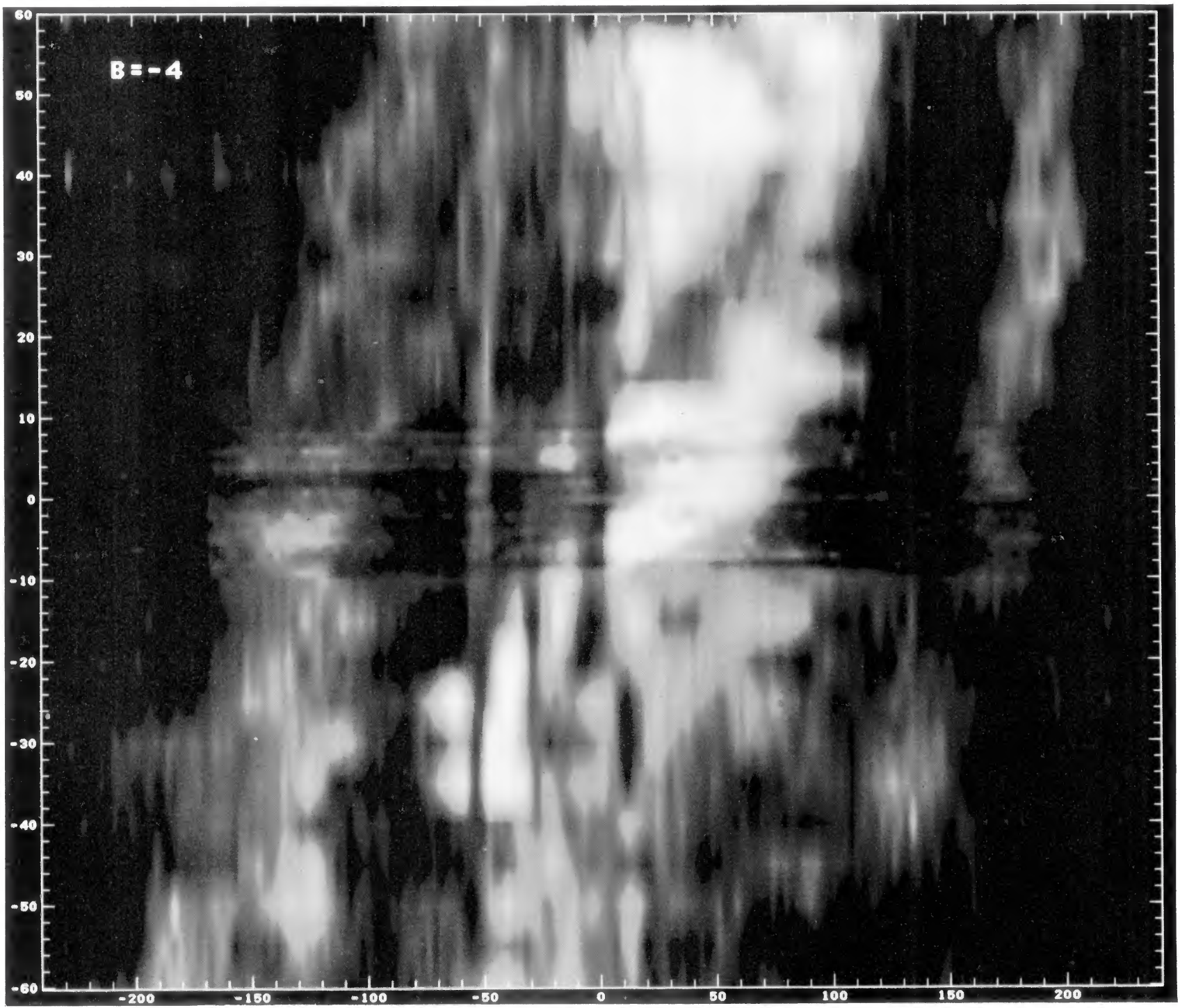

FIG. 5.-Gray-scale representation of ${ }^{12} \mathrm{C}^{16} \mathrm{O}$ emission and absorption intensities in longitude-velocity coordinates at $\Delta b=-4^{\prime}$, $-60^{\prime} \leq \Delta l \leq 60^{\prime}$. At $|\Delta l| \leq 10^{\prime}$, the observations are separated in longitude by $1^{\prime}$; elsewhere by $5^{\prime}$.

Liszt et al. (see page 39) 